\title{
Spectral attenuation of sound in dilute suspensions with nonlinear particle relaxation
}

\author{
Max Kandula \\ ASRC Aerospace, NASA Kennedy Space Center, FL 32899, USA \\ max.kandula-1@ksc.nasa.gov
}

\begin{abstract}
Previous studies on the sound attenuation in particle-laden flows under Stokesian drag and conduction-controlled heat transfer have been extended to accommodate the nonlinear drag and heat transfer. It has been shown that for large particle-to-fluid density ratio, the particle Reynolds number bears a cubic relationship with $\omega \tau_{d}$ (where $\omega$ is the circular frequency and $\tau_{d}$ the Stokesian particle relaxation time). This dependence leads to the existence of a peak value in the linear absorption coefficient occurring at a finite value of $\omega \tau_{d}$. Comparison of the predictions with the test data for the spectral attenuation of sound with water injection in a perfectly expanded supersonic air jet shows a satisfactory trend of the theory accounting for nonlinear particle relaxation processes.
\end{abstract}

PACS numbers: $43.20 . \mathrm{Hq}, 43.50 . \mathrm{Nm}, 43.50 . \mathrm{Gf}$

\section{Introduction}

Sound attenuation in fluids, representing the dissipation of acoustic energy from a sound wave, occurs through a number of physical processes involving molecular viscosity, thermal conductivity, and other dissipative or relaxation processes. ${ }^{1-4}$ In all these absorption mechanisms, acoustic energy is converted into thermal energy, and other mechanisms deflect or scatter 
acoustic energy. ${ }^{1}$ Stokes ${ }^{5}$ developed the first successful theory of sound absorption due to the effect of molecular viscosity of the fluid (internal friction). Helmholtz ${ }^{6}$ theoretically investigated the effect of viscosity on the sound attenuation in a circular tube, considering the effect of friction near solid boundaries. Kirchhoff ${ }^{7}$ theoretically treated sound attenuation due to the viscosity and heat conduction effects in an unbounded fluid medium and in narrow tubes. The so-called classical sound absorption in fluids includes Stokes' viscous contribution and Kirchhoff's thermal conduction contribution to attenuation in unbounded fluids.

When a fluid contains inhomogeneities such as suspended particles (solid particles, drops and bubbles) additional viscous and heat conduction losses occur in the immediate neighbourhood of the suspended particles. ${ }^{1-3,8} \mathrm{~A}$ comprehensive review of the physics and scientific history of acoustic interactions with particulate mixtures is provide by Challis et al. ${ }^{9}$ Referring to Fig. 1, the acoustic intensity $I$ of a plane wave propagating through an absorbing medium is expressed by

$$
I=I_{0} e^{-\alpha_{i} x}
$$

where $x$ is the distance traversed, $I_{0}$ the intensity at $x=0$, and $\alpha_{i}$ the intensity attenuation coefficient for the medium. The quantity $\alpha_{i}$ depends on viscosity, thermal conductivity, and other factors such as molecular relaxation.

Sound propagation in aerosols and fog has been studied experimentally and theoretically by several investigators. Sewell, ${ }^{10}$ in his pioneering work, theoretically considered the attenuation of sound in a viscous medium containing suspended cylindrical or spherical particles (obstacles) with perfectly rough surfaces. With the aid of a scattering formulation (theory), Sewell predicted the attenuation of sound by rigid particles suspended in a gas, assuming that the particles are immovable. Epstein, ${ }^{11}$ in a theoretical treatment of the attenuation of sound by spherical particles suspended in liquids or gases, derived and extended Sewell's result by permitting the particles to 
move. Epstein and Carhart ${ }^{12}$ additionally considered heat conduction effects, and found that for fogs the effects of viscosity and heat conduction are both important, and approximately additive. Their formulation was extended by Allegra and Hawley ${ }^{13}$ to include liquid-liquid as well as liquid-solid systems (with a different model for each case).

The effect of transport processes on the attenuation and dispersion of sound in aerosols have also been reported by Soo, ${ }^{14}$ accounting for nonstationary effects including the Basset history (effect of particle acceleration on viscous drag) and added mass terms. Basset history and added mass terms were included in an elegant coupled phase formulation by Harker and Temple. $^{15}$ Coupled phase effects were also treated by Evans and Attenborough ${ }^{16,17}$ in an extension to the work of Harker and Temple ${ }^{15}$ to incorporate thermal conduction. Nonstationary effects, which become important at low particle Reynolds numbers, are further investigated by Gumerov ${ }^{18}$. Chow ${ }^{19}$ considered the attenuation of sound in dilute emulsions and suspensions with viscous dissipation and thermal conduction, and additionally included the effect of surface tension, which was shown to be important in the presence of bubbles (and negligible for systems with solid particles or liquid droplets).

Temkin and Dobbins, ${ }^{20}$ in their classical work, theoretically considered particle attenuation and dispersion of sound in a manner which illustrates explicitly the relaxation character of the problem. For rigid particles, the linear droplet drag and heat transfer are respectively obtained from

$$
\begin{gathered}
F_{p}=6 \pi \mu_{g}\left(u_{p}^{\prime}-u_{g}^{\prime}\right) \\
Q_{p}=2 \pi d_{p} k_{g}\left(T_{p}^{\prime}-T_{g}^{\prime}\right)
\end{gathered}
$$

which correspond to the zero droplet Reynolds number limit $\left(\operatorname{Re}_{p} \rightarrow 0\right)$, where

$$
\operatorname{Re}_{p}=\rho_{g}\left|u_{g}-u_{p}\right| d_{p} / \mu_{g}
$$


In the above, $u$ is the velocity, $T$ the temperature, $\rho$ the density, $\mu$ the dynamic viscosity, $k$ the thermal conductivity, and $d$ the particle diameter. The subscripts $g$ and $p$ stand respectively for the gas and the particle, and the primes denote fluctuations from the mean. The properties $\rho_{g}, \mu_{g}, k_{g}$ and $\rho_{p}$ refer to the mean values. According to Temkin and Dobbins, ${ }^{20}$ Stokes linear drag law can be justified for $0 \leq \omega \tau_{d} \approx 1$, provided that $\rho_{g} / \rho_{p} \ll<1$ and $\left(\omega d_{p}^{2} / 8 v_{g}\right)^{1 / 2} \ll 1$

Recent data ${ }^{21}$ on sound attenuation in supersonic air jets containing suspended water droplets reveal that the linear absorption coefficient displays a spectral peak. The particulate relaxation models for the sound attenuation are all based on Stokes drag (linear drag law) and pure conduction limit (linear heat transfer), and do not account or explain this attenuation behaviour. This article, primarily based on Ref. 22, attempts to investigate this attenuation behaviour by considering nonlinear drag and heat transfer laws applicable to relatively largesized droplets.

\section{Analysis for sound attenuation}

The present analysis extends the work of Temkin and Dobbins ${ }^{20}$ for dilute suspensions to accommodate the nonlinear drag and heat transfer laws, which become important at high particle Reynolds numbers $\operatorname{Re}_{p}$ and at high frequencies. Only sound attenuation is considered here, with sound dispersion excluded from consideration.

\subsection{Nonlinear particle drag and heat transfer}

The theory of Temkin and Dobbins ${ }^{20}$ or dilute solutions is applicable at low mass concentrations $C_{m}=n_{0} m_{p} / \rho_{g} \ll<$, where $n_{0}$ refers to the mean number of particles per unit volume of mixture, and $m_{p}$ the mass of one particle. Without any loss of generality the 
attenuation of sound for large particle Reynolds numbers with nonlinear particle relaxation may be expressed as follows:

$$
\bar{\alpha}=\left(\frac{c_{0} \alpha}{C_{m} \omega}\right)=\frac{\omega \tau_{d 1}}{1+\omega^{2} \tau_{d 1}^{2}}+(\gamma-1)\left(\frac{c_{p p}}{c_{p g}}\right) \frac{\omega \tau_{t 1}}{1+\omega^{2} \tau_{t 1}^{2}}
$$

In the above the quantity $\bar{\alpha}$ refers the attenuation per unit frequency per unit mass fraction, $\alpha$ the linear absorption coefficient or the amplitude attenuation coefficient (imaginary part of the wavenumber), $c_{p}$ the specific heat, $c_{0}$ the speed of sound in the gas phase, and $\gamma$ the isentropic exponent (specific-heat ratio). Note that $\alpha=\alpha_{i} / 2$.

The relaxation times $\tau_{d 1}$ and $\tau_{t 1}$ correspond to those under nonlinear drag conditions (generally representative of large-sized particles). They are related to the relaxation times $\tau_{d}$ and $\tau$, by the relations

where

$$
\begin{gathered}
\tau_{d 1}=\tau_{d} \psi_{1}\left(\operatorname{Re}_{p}\right), \tau_{t 1}=\tau_{t} \psi_{2}\left(\operatorname{Re}_{p}\right) \\
\psi_{1}\left(\operatorname{Re}_{p}\right)=C_{D 1} / C_{D}, \psi_{2}\left(\operatorname{Re}_{p}, \operatorname{Pr}\right)=N u_{1} / N u
\end{gathered}
$$

with $C_{D 1}$ standing for the nonlinear drag coefficient, and $N u_{1}$ for the nonlinear heat transfer. In the above,

and

$$
\begin{gathered}
\tau_{d}=d_{p}^{2} \rho_{p} /\left(18 \mu_{g}\right) \\
\tau_{t}=\frac{m_{p} c_{p p}}{2 \pi d_{p} k_{g}}=\frac{\operatorname{Pr} c_{p p} d_{p}^{2} \rho_{p}}{12 \mu_{g} c_{p g}}=\left(\frac{3}{2}\right)\left(\frac{c_{p p}}{c_{p g}}\right) \operatorname{Pr} \tau_{d}
\end{gathered}
$$

where $\operatorname{Pr}=c_{p g} \mu_{g} / k_{g}$ stands for the Prandtl number of gas. Physically the quantity $\tau_{d i}$ is a measure of the inability of the particles to follow (respond to) the fluctuations in the fluid motion. ${ }^{3}$ Likewise, the quantity $\tau_{t 1}$ is a measure of the thermal response of the particles to follow the fluctuations in the temperature of the fluid. 
The drag coefficient and the Nusselt number in Eq. (3c) are defined by

$$
C_{D}=2 F_{D} /\left(\rho_{g} \frac{\pi}{4} d_{p}^{2} u_{g}^{2}\right), \quad N u=h_{g} d_{p} / k_{g}
$$

where $F_{D}$ is the particle drag force, and $h_{g}$ the gas-droplet convective heat transfer coefficient. A good approximation (curve fit) for the drag coefficient $C_{D 1}$ is recommended as ${ }^{23}$

$$
C_{D 1}=\frac{24}{\operatorname{Re}_{p}}+\frac{6}{1+\sqrt{\operatorname{Re}_{p}}}+0.4
$$

The first term on the RHS of Eq. (6) is the Stokesian drag coefficient defined by

$$
C_{D}=24 / \operatorname{Re}_{p}
$$

which is valid for particle Reynolds numbers less than about one. Eqs. (5) and (6) yield an expression for $\psi_{1}$ as

$$
\psi_{1}\left(\operatorname{Re}_{p}\right)=1+\frac{\operatorname{Re}_{p}}{24}\left(\frac{6}{1+\sqrt{\operatorname{Re}_{p}}}+0.4\right)
$$

With regard to the particle heat transfer, a good correlation for heat transfer (by conduction and convection) is expressed by the well-known Ranz-Marshall correlation ${ }^{24}$

$$
N u_{1}=2+0.6 \operatorname{Re}_{p}^{0.5} \operatorname{Pr}^{0.33}
$$

In the pure conduction limit, we have $N u=2$, so that the function $\psi_{2}$ in Eq. (3c) becomes

$$
\psi_{2}=N u_{1} / N u=1+0.3 \operatorname{Re}_{p}^{0.5} \operatorname{Pr}^{0.33}
$$

It now remains to find a relation for the droplet Reynolds number $\operatorname{Re}_{p}$ in terms of the density ratio and the particle relaxation time. 


\subsection{Droplet Reynolds number}

The determination of particle Reynolds number required in the evaluation of the functions $\psi_{1}$ and $\psi_{2}$ in Eqs. (8) and (10) respectively is exceedingly complex. There exists relatively little information on the dependence of particle Reynolds number on the particle characteristics in twophase flows. A study of turbulent diffusion of droplets in a gaseous medium ${ }^{25,26}$ indicates a plausible relationship of the form

$$
\operatorname{Re}_{p}=f\left(\rho_{g} / \rho_{p}, \omega \tau_{d}\right)
$$

Since Eq. (3a) is applicable to large particle to fluid density ratios, we postulate here that the particle Reynolds number depends only on the particle relaxation time, and is independent of the particle to fluid density ratio: that is

$$
\operatorname{Re}_{p}=f\left(\omega \tau_{d}\right)
$$

The physical basis for this supposition may be justified as follows. Particles with a smaller relaxation time are able to respond better to the fluctuations in the fluid motion, which leads to reduced particle relative velocity, and thus a reduced particle Reynolds number.

In the present investigation, the following power law relation is proposed such that a peak in the linear absorption coefficient (as indicated by the measurements) is realized (an exponent of 2.5 or greater for the relaxation time ensures this peak):

$$
\operatorname{Re}_{p}=c\left(\omega \tau_{d}\right)^{3}
$$

The adjustable constant $c$ is determined from a correlation of the theory with the test data. A value of $c=10$ is found to be satisfactory based on the data of Norum ${ }^{21}$ for water droplets in a supersonic air jet. 


\section{Results and comparison}

\subsection{Effect of Nonlinear Particle Relaxation}

The effect of nonlinear particle relaxation on the absorption coefficient per unit frequency is demonstrated in Fig. 2a for comparison with the theory of Temkin and Dobbins ${ }^{20}$ for dilute concentrations. The results suggest that below $\omega \tau_{d} \approx 0.7$ the nonlinear particle relaxation effects are seen to be unimportant. Beyond $\omega \tau_{d} \approx 0.7$, the nonlinear theory departs from the linear theory.

Fig. $2 \mathrm{~b}$ shows a comparison of the predicted linear spectral attenuation of sound under nonlinear particle relaxation. The theoretical result by Temkin and Dobbins ${ }^{20}$ for dilute concentrations is also presented for a comparison. The theory suggests that at high frequencies the linear absorption coefficient predicted by the nonlinear theory decreases with increasing frequency in accordance with experimental trend for large particle sizes.

\subsection{Comparison with experimental data}

A direct comparison of the present theory with the measured spectral attenuation by Norum ${ }^{21}$ for water droplets in a perfectly expanded (jet exit pressure equals the ambient pressure; thus shockfree) supersonic jet is displayed in Fig. 3. The data correspond to hot supersonic jet of air from a convergent-divergent (CD) nozzle operation at a jet total temperature $T_{t}=867 \mathrm{~K}$, and a jet exit Mach number $M_{j}=1.45$. The jet Mach number is defined as $M_{j}=u_{j} / c_{j}$, where the subscript $j$ refers to the nozzle exit conditions. The mass flow rate (maximum considered) of water to that of the jet is about 0.85 . The angle $\theta$ is measured from the jet inlet axis. The jet exit Reynolds number $\operatorname{Re}_{j 1}=u_{j} d_{j} / \mu_{g j}$ is about $1.3 \times 10^{6}$, where $d_{j}$ is the jet exit diameter. At this condition, supersonic turbulent jet mixing noise dominates upstream noise radiation, and Mach wave radiation dominates the downstream noise radiation. In the data, water is injected at $45 \mathrm{deg}$. The 
data include spectra measured at angles of $45 \mathrm{deg}, 90 \mathrm{deg}$ and $135 \mathrm{deg}$, thus highlighting directivity effects.

From the measured $\triangle \mathrm{SPL}$ (reduction in Sound Pressure Level) at a given frequency due to water injection, the linear absorption coefficient is deduced as

$$
\alpha / \alpha_{\max }=\Delta \mathrm{SPL} / \Delta \mathrm{SPL} \max
$$

where the subscript refers to the peak spectral reduction. The comparisons suggest that the proposed theory based on the nonlinear particle relaxation processes satisfactorily describes the measurements for the spectral attenuation of sound, indicating a spectral peak. The inclusion of directionality effect (dependence on the angle of observation) on the spectral absorption is beyond the scope of the present work.

It should be remarked, however, that the data on supersonic jet noise considered here for comparison are not directly pertinent to plane waves of sound for which the theory has been developed. Since noise from turbulent jets may be regarded as a superposition of plane waves of differing frequency, the absolute values of the absorption coefficient in the test data could be different from that expressed by the theory. In this connection, the reduction of turbulent jet mixing noise reduction by water injection was theoretically considered by the author in Ref. 27 in conjunction with the scaling laws proposed for jet noise ${ }^{28}$. Finally, the effect of droplet

evaporation on the spectral attenuation of sound, as indicated by the theory of Marble et al. ${ }^{29}$ is left out of account in the present analysis.

\section{Conclusion}

The theory proposed here for sound attenuation in dilute suspensions with nonlinear particle drag and heat transfer is shown to satisfactorily represent the test data for noise reduction with water droplets suspended in a supersonic jet. It is found that the nonlinear particle relaxation processes are primarily responsible for reduction in the linear absorption coefficient at high frequencies. 


\section{Acknowledgments}

The author is grateful to the reviewer for helpful suggestions including several important references pertinent to suspension acoustics. Dr. Thomas Norum of NASA Langley Research Center kindly provided the spectral data for comparison. Thanks are also due to Dr. Paul Schallhorn (Chief, Environments and Launch Approval Branch) and Leonard Duncil of the Launch Services Program at NASA Kennedy Space Center for partial financial support of this work. 


\section{References and links}

${ }^{1}$ L.E. Kinsler, A.R. Frey, A.B. Coppens, and J.V. Sanders, Fundamentals of Acoustics (JohnWiley and Sons, New York, 1982).

${ }^{2}$ A.D. Pierce, Acoustics- An introduction to Physical Principles and Applications (The Acoustical Society of America, 1994).

${ }^{3}$ S. Temkin, Elements of Acoustics (The Acoustical Society of America, 2001).

${ }^{4}$ J.W.S. Rayleigh, The Theory of Sound, Vol. 2 (Dover, New York, 1945).

${ }^{5}$ G.G. Stokes, "On the theories of internal friction of fluids in motion", Trans. Cambridge Phil. Soc. 8, 287-305 (1845).

${ }^{6} \mathrm{H}$. von Helmholtz, "On the influence of friction in the air on sound motion," Verhandl. Naturhist. Med. Ver. Heidelberg 3, 16-20 (1863); reprinted in Wissenschftliche Abhandlungen, Barth, Leipzig 1, 383-387 (1882).

${ }^{7} \mathrm{G}$. Kirchhoff, "On the influence of heat conduction in a gas on sound propagation," Ann. Phys. Chem. (5) 134, 177-193 (1868).

${ }^{8}$ S. Temkin, Suspension Acoustics (Cambridge University Press, Cambridge, 2005).

${ }^{9}$ R.E. Challis, M.J.W. Povey, M.L. Mather, and A.K. Holmes, "Ultrasound techniques for characterizing colloidal dispersions," Reports on Progress in Physics 68, 1541-1637 (2005). ${ }^{10}$ C.J.T. Sewell, "On the extinction of sound in a viscous atmosphere by small obstacles of cylindrical and spherical form," Phil. Trans. Roy. Soc. London (A) 210, 239-270 (1910).

${ }^{11}$ P.S. Epstein, "On the absorption of sound waves in suspensions and emulsions," Theodore von Karman Anniversary Volume, p. 162 (1941) 
${ }^{12}$ P.S. Epstein and R.R. Carhart, "The absorption of sound in suspensions and emulsions, $\mathrm{I}$. Water fog in air," J. Acous. Soc. Am. 25, 553-565 (1953)

${ }^{13}$ J.R. Allegra and S.A. Hawley, "Attenuation of sound in suspensions and emulsions: Theory and experiments," J. Acous. Soc. Am. 51, 1545-1564 (1972).

${ }^{14}$ S.L. Soo, "Effect of transport properties on attenuation and dispersion in aerosols," $J$. Acoust. Soc. Am. 32, 943-946 (1960).

${ }^{15}$ A.H. Harker and J.A.G. Temple, "Velocity and attenuation of ultrasound in suspensions of particles in fluid," J. Phys. D: Appl. Phys. 21, 1576-1588 (1988).

${ }^{16}$ J.M. Evans and K. Attenborough, "Coupled phase theory for sound propagation in emulsions," J. Acous. Soc. Am. 102, 278-282 (1997).

${ }^{17}$ J.M. Evans and K. Attenborough, "Sound propagation in concentrated emulsions" Comparison of coupled phase model and core-shell model," J. Acous. Soc. Am. 112(5), 1911 1917 (2002).

${ }^{18}$ N.A. Gumerov, A.I. Ivandaev, and R.I. Nigmatulin, "Sound waves in a monodisperse gasparticle or vapor-droplet mixtures," J. Fluid Mech. 193, 53-74 (1988).

${ }^{19}$ J.C.F. Chow, "The attenuation of acoustic waves in dilute emulsions and suspensions," $J$. Acoust. Soc. Am. 36, 2395-2401 (1964).

${ }^{20}$ S. Temkin and R.A. Dobbins, "Attenuation and dispersion of sound by particulate relaxation processes," J. Acoust. Soc. Am. 40, 317-324 (1966).

${ }^{21}$ T.D. Norum, "Reductions in multi-component jet noise by water injection,"AIAA-20042976, $10^{\text {th }}$ AIAA/CEAS Aeroacoustics Conference, Manchester, Great Britain, May (2004).

${ }^{22} \mathrm{M}$. Kandula and M. Lonergan, "Spectral attenuation of sound in dilute suspensions with nonlinear particle relaxation,"Acoustics'08 Paris Conference, Paris, France, June 29 - July 4 (2008). 
${ }^{23}$ F.M. White, Viscous Fluid Flow, $2^{\text {nd }}$ ed. (McGraw-Hill, New York, 1991).

${ }^{24}$ W.E. Ranz, W.P. Marshall, Chemical Eng. Prog. 48, 141, p. 173 (1952).

${ }^{25} \mathrm{~K}$. Mastanaiah, "Experimental and theoretical investigation of droplet deposition and heat transfer in two phase turbulent flow through a vertical tube," Ph.D. thesis, University of Illinois, Chicago (1980).

${ }^{26}$ E.N. Ganic and K. Mastanaiah, "Investigation of droplet deposition from a turbulent gas stream," Int. J. Multiphase Flow 7, 401-411 (1981)

${ }^{27} \mathrm{M}$. Kandula and M. Lonergan, "Effective jet properties for the estimation of turbulent jet noise reduction by water injection," AIAA-2007-3645, $10^{\text {h }}$ AIAA/CEAS Aeroacoustics Conference, Rome, Italy, May (2007); also submitted in revised form to AIAA J., 2008.

${ }^{28} \mathrm{M}$. Kandula, "On the scaling laws and similarity spectra for jet noise in subsonic and supersonic flow," Int. J. Acoustics and Vibration 13(1), 3-16 (2008).

${ }^{29}$ F.E. Marble and S.M. Candel, "Acoustic attenuation in fans and ducts by vaporization of liquid droplets," AIAA J. 13, 634-639 (1975). 


\section{Figure Captions}

Fig. 1 Attenuation of a plane sound wave in a gas-droplet mixture.

Fig. 2a (color online) Predicted absorption coefficient per unit frequency with nonlinear particle relaxation processes.

Fig. $2 b$ (color online) Predicted linear absorption coefficient with nonlinear particle relaxation processes.

Fig. 3 (color online) Comparison of the predictions for the linear absorption coefficient with test data of Norum ${ }^{21}$. 
Fig. 1 Attenuation of a plane sound wave in a gas-droplet mixture. 


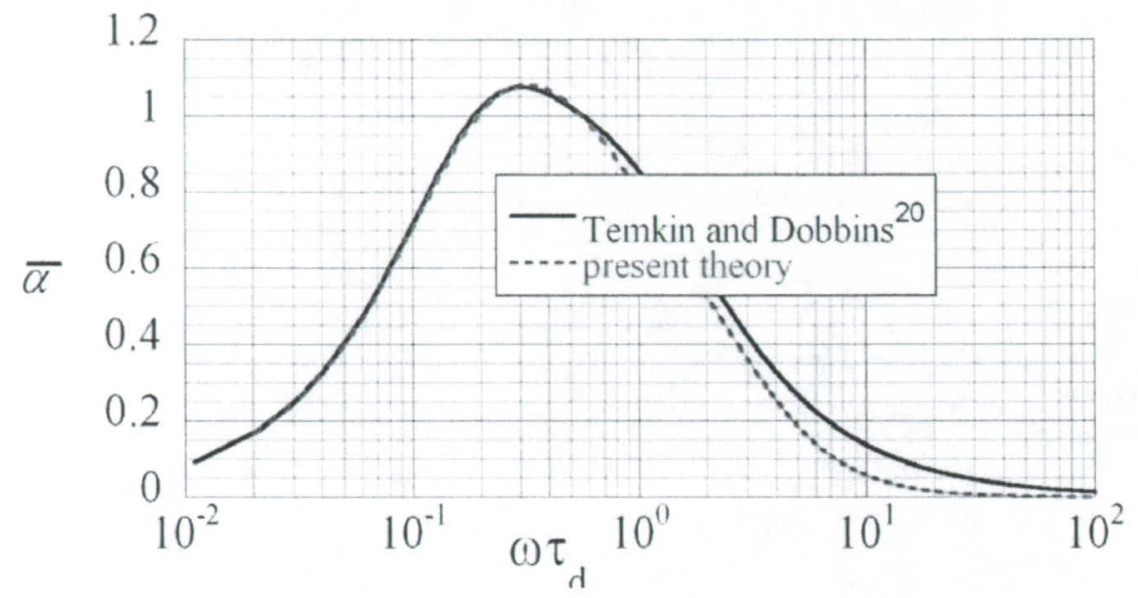

Fig. 2a Predicted absorption coefficient per unit frequency with nonlinear particle relaxation processes. 


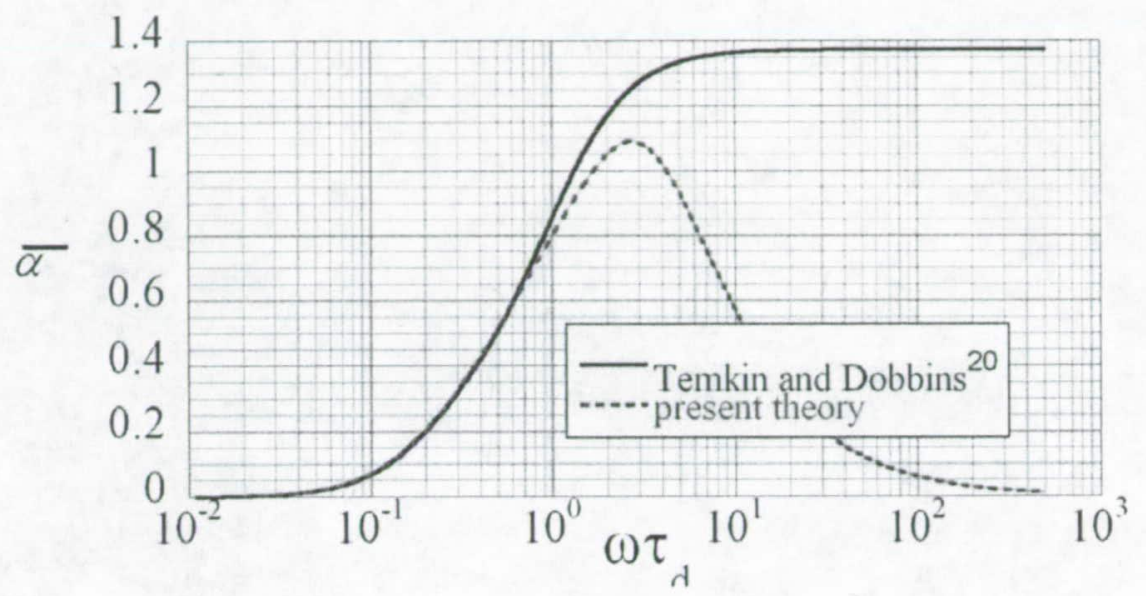

Fig. 2b Predicted linear absorption coefficient with nonlinear particle relaxation processes. 


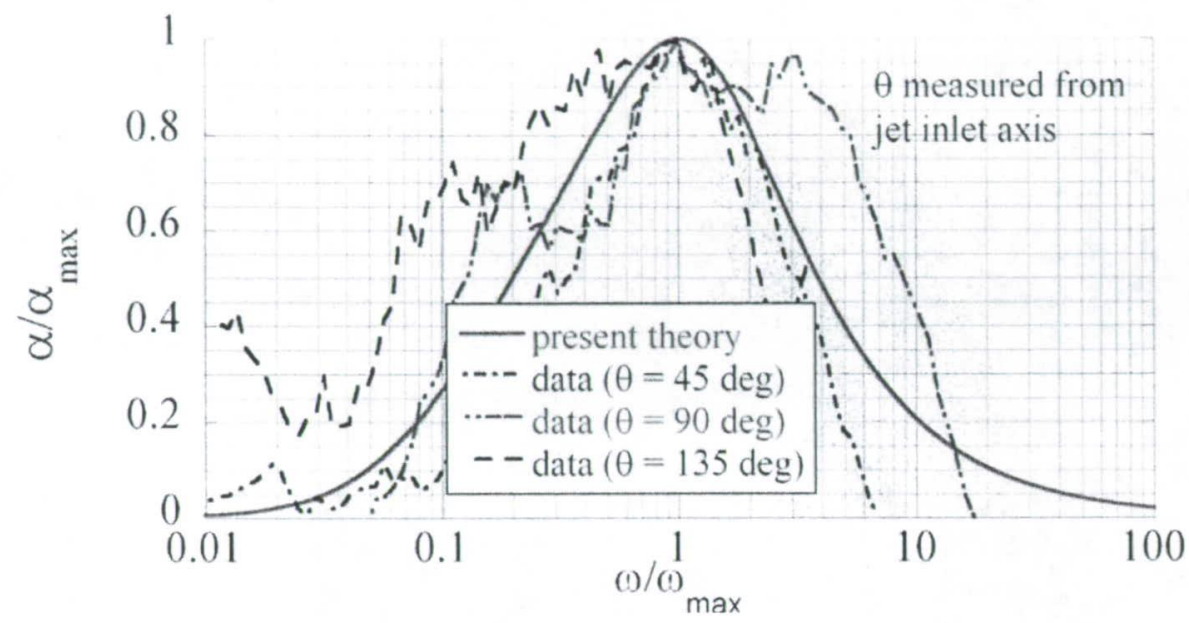

Fig. 3 Comparison of the predictions for the linear absorption coefficient with test data of Norum ${ }^{21}$. 
The public reporting burden for this collection of information is estimated to average 1 hour per response, including the time for reviewing instructions, searching existing data sources, gathering and maintaining the data needed, and completing and reviewing the collection of information. Send comments regarding this burden estimate or any other aspect of this collection of information, including suggestions for reducing this burden, to Department of Defense. Washington Headquarters Services, Directorate for Information Operations and Reports (0704-0188), 1215 Jefferson Davis Highway, Suite 1204, Arlington, VA 22202-4302. Respondents should be aware that

notwithstanding any other provision of law, no person shall be subject to any penalty for failing to comply with a collection of information if it does not display a currently valid OMB control number.

PLEASE DO NOT RETURN YOUR FORM TO THE ABOVE ADDRESS.

\section{\begin{tabular}{l|l} 
1. REPORT DATE (DD-MM-YYYY) & 2. REPORT TYPE
\end{tabular} \\ Journal Article}

\section{TITLE AND SUBTITLE}

Spectral Attenuation of Sound in Dilute Suspensions with Nonlinear Particle Relaxation
3. DATES COVERED (From - To) 5a. CONTRACT NUMBER

NAS 10-03006

\section{5b. GRANT NUMBER}

5c. PROGRAM ELEMENT NUMBER

5d. PROJECT NUMBER

5e. TASK NUMBER

5f. WORK UNIT NUMBER

6. AUTHOR(S)

Kandula, Max

\section{PERFORMING ORGANIZATION} REPORT NUMBER

7. PERFORMING
ASRC Aerospace

ASRC-5211

Kennedy Space Center, FL 32899

9. SPONSORING/MONITORING AGENCY NAME(S) AND ADDRESS(ES)

National Aeronautics and Space Administration

$\mathrm{NE}$

Kenndey Space Center, FL 32899

10. SPONSORING/MONITOR'S ACRONYM(S)

NASA KSC

11. SPONSORING/MONITORING REPORT NUMBER

\section{DISTRIBUTIONIAVAILABILITY STATEMENT}

\section{SUPPLEMENTARY NOTES}

\section{ABSTRACT}

Previous studies on the sound attenuation in particle-laden flows under Stokesian drag and conduction-controlled heat transfer have been extended to accomodate the nonlinear drag and heat transfer. It has been shown that for large particle-to-fluid density ratio, the particle Reynolds number bears a cubic relationship with wtd (where $w$ is the circular frequency and td the Stokesian particle relaxation time). This dependence leads to the existence of a peak value in the linear absorption coefficient occurring at a finite value of wtd. Comparison of the predictions with the test data for the spectral attenuation of sound with water injection in a perfectly expanded supersonic air jet shows a satisfactory trend of the theory accounting for nonlinear particle relaxation processes.

\section{SUBJECT TERMS}

spectral attenuation, nonlinear particle relaxation, sound

\begin{tabular}{|c|c|c|}
\hline \multicolumn{2}{|l|}{ 16. SECURITY CLASSIFICATION OF: } \\
\hline a. REPORT & b. ABSTRACT & c. THIS PAGE \\
& & \\
\hline
\end{tabular}

\section{LIMITATION OF ABSTRACT}

18. NUMBER

OF

PAGES

19b. NAME OF RESPONSIBLE PERSON

Max Kandula

19b. TELEPHONE NUMBER (Inc/ude area code)

18

(321) 867-4456 